\title{
Finite Element Analysis of the Effect on Edge Distance of the Tensile Bearing Capacity of Embedded Hanging Parts
}

\author{
Xian Hong MENG ${ }^{1}$, Wei LIU ${ }^{1, a}$ \\ 1 School of Civil Engineering, Shenyang Jianzhu University
}

\begin{abstract}
In order to explore the trend of tensile bearing capacity of embedded hanging parts when change the edge distance. Based on the finite element analysis software ABAQUS, the four simulation model was established. The buried depth and strength of concrete remain unchanged, but the edge distance was gradient change. By the load - displacement curve of every model known, the greater the edge distance, the greater the bearing capacity. When the edge distance reaches 1.5 times buried depth, the effect of increasing edge distance for improving the bearing capacity will be impaired.
\end{abstract}

\section{Overviews}

The embedded hanging parts are special steel components, which are applied to the production, lifting and transportation of prefabricated structures. With the development of prefabricated structure, people gradually realize that the importance of embedded hanging parts of production, testing, application.

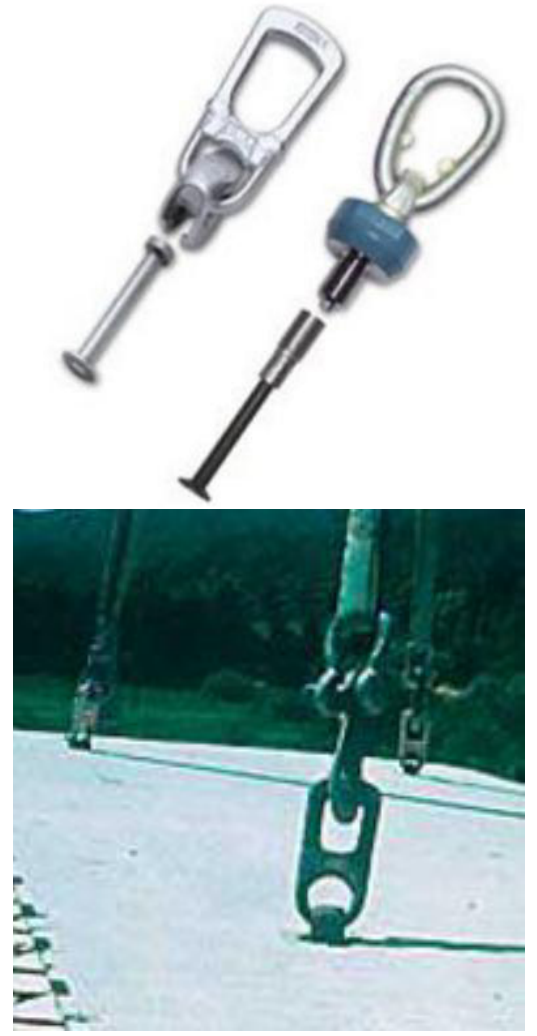

Figure 1 Embedded hanging parts
The traditional embedded hanging parts is made by the rings, Which is fixed at the special position before prefabricated parts are lifted. It will be the reliable connection between concrete and sling unless the strength of concrete reach its $70 \%$ of its design strength. People will cut the Exposed portion of the rings with angle grinder and grinding wheel, when the prefabricated parts are installed. But there is no doubt that the bar at the incisions will affect the beautiful of structure and the durability in lifetime. The rings is required that the burial depth over 30 times its diameter when it is used to lifting, in order to prevent it is pulled from the concrete. This way also waste a lot of steel to some extent. So, the ring is eliminated in the construction industry.

In order to meet the development of Prefabricated buildings, the requirements to embedded hanging parts will Increase year by year. The size of current embedded hanging parts have been optimized, and their height less than $100 \mathrm{~mm}$, diameter less than $30 \mathrm{~mm}$, decreasing the amount of the steel effectively. It's possible that the maximal lifting load can reach 50t with the high strength steel. Their exposed portions always are designed as ring, and the connection between the buried portion and sling can be finished by the threads. So, it makes sure the sling rotation, tilt freely in lifting, decreasing the extrusion and shear between embedded hanging parts and its surrounding concrete.

Embedded hanging parts stem from Europe and the United States, and relevant specifications have been established for guiding its engineering application in foreign, such as European standardETAG001 Annex A and Annex $C$, American codeACI318 Annex D. The specifications define the failure mode, calculating capacity,parameters modification , but the control standards, loading system, unit conversion in foreign codes are different from them in home. Thus, the value

\footnotetext{
${ }^{a}$ Wei Liu:1280299968@qq.com
} 
from foreign codes only has reference significance, which don't consider as the yardstick of measuring that whether embedded hanging parts are safe usage or not. In home, we have almost no relevant specification for embedded hanging parts, only the modification method of calculating Tensile bearing capacity of embedded hanging parts are mentioned by Technical Specification for Post-installed Fastenings in Concrete Structures, when it comes to define the Anchor bolt. In addition, a mass of relevant research define Four kinds of failure modes: anchor bolt steel fracture ${ }^{[1-3]}$, Anchor bolt pulled up failure, Concrete vertebral failure by tensile, Concrete fracturing failure. However, the embedded hanging parts' transmit strength way, Stress analysis are different from Anchor bolts. So, the tensile bearing capacity of embedded hanging parts can't be calculated accurately according to the theoretical calculation method in Anchor bolt codes. It's similar for the way of embedded hanging parts, Anchor bolt and Planting bar between concrete, thus, the existing Anchor bolt and Planting bar research result can be learned when we research the effect of edge distance for embedded hanging parts. Wu Guifang ${ }^{[4]}$ researched the four kinds of failure model mentioned in ACI318 Annex D, and come to a conclusion that it's no necessity to consider the side split of concrete, when the edge distance more than 4.8 times burial depth. The research of Yuan Tingpeng ${ }^{[5]}$ indicated that the factors that effecting the tension capacity of Planting bar include Strength of concrete, burial depth, edge distance, spacing and so on, put forward the tension capacity empirical formula of Group of reinforcement, and involving the correction factor of every factors.

At present, the manufacturers of embedded hanging parts always provide the Nominal load to users, and it's also called safety load. Which is a safety value without considering the reduction effect of edge distance, burial depth and other factors. But it's common that embedded hanging parts have no enough safety edge distance in practical engineering application. The tension capacity must less than safety load in that case, and the reduction factor offered by engineers through the their experience. Besides, safety load always have no enough safety factor, Burying the hidden trouble for safety construction. From what has been discussed above, it is necessary to study the edge distance of embedded hanging parts.

From what has been discussed above, it is necessary to consider the effect of edge distance to tensile bearing capacity . But a great of time, manpower and material will be consumed at experimental stud, and there are many factors to affect the tensile bearing capacity of embedded hanging parts, which can't got from the experimental stud. However, the finite element analysis can solve the question by changing the factors, observing the analysis result easily. Besides, this way also can guide the following experiment, including the gradient change of factors, Size optimization, loading scheme and so on.

\section{2 finite element analysis}

\subsection{Model design}

Based on the finite element analysis software ABAQUS, model is established, including the concrete and embedded hung part. There is no bar inside the concrete, and embedded hanging parts' material are Q345, Model size is shown in figure 2 .

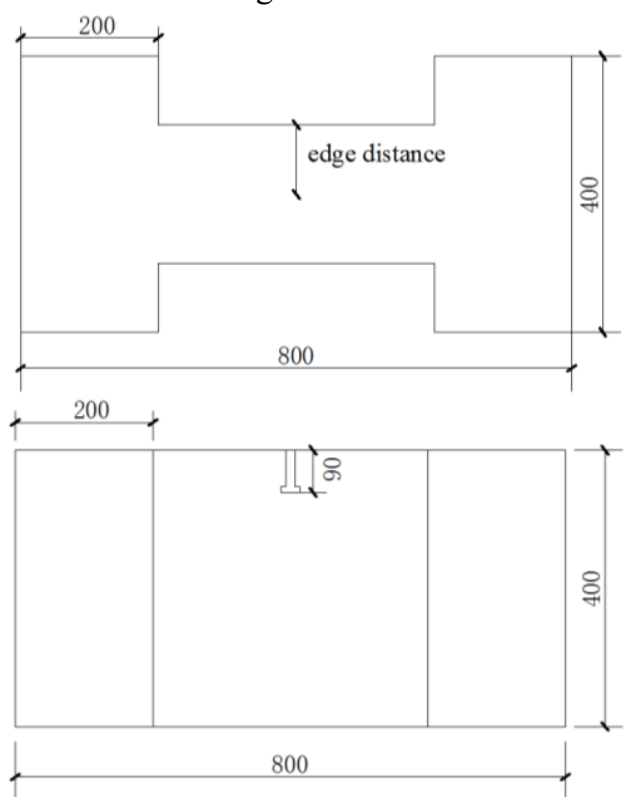

Figure 2 Model size

The model of embedded hung part in finite element analysis comes from a company product prototype. Its diameter is $12 \mathrm{~mm}$, effective depth is $90 \mathrm{~mm}$, safety load is $62.5 \mathrm{kN}$. According to the provisions of Anchor bolt specification at home and abroad, it's no necessary to consider the reduction effect of edge distance, if the edge distance more than 1.5 times effective depth. Thus, the critical edge distance is defined as $135 \mathrm{~mm}$ in this research. In order to explore the effect of edge distance, author designs four models that their edge distance are gradient change. Main parameters are shown in Table 1.

Table 1. Main parameters

\begin{tabular}{|c|c|c|c|c|}
\hline Model number & Y1 & Y2 & Y3 & Y4 \\
\hline Edge distance $(\mathrm{mm})$ & 50 & 100 & 135 & 150 \\
\hline
\end{tabular}

The Table 1 shows that, Y3's edge distance are critical, Y2's and Y1's edge progressively decreases. Y4 has no effect of edge distance.

\subsection{Finite element simulation}

\subsubsection{Establishing the model}

Establishing the model according to the model size before finished at previous step, the research focus on effcet of edge distance for tensile bearing capacity when concrete appear vertebral failure. So, we hold that there is no relative slip between embedded hanging parts and concrete, and the interface between them has enough cohesive force. Thus, the constraint between them is defined as embedded.

\subsubsection{Define the material}


The strength grade of concrete is C20, and the constitutive relation is concrete damaged plasticity model. Embedded hanging parts' material is Q345, established as ideal elastic-plastic model.

\subsubsection{Load and Constraint}

The load and constraint of models are shown in figure 3 , two flanks of the concrete are on the surface Fixed constraint, and loading vertically at exposed end of embedded hanging parts. We can get the Ultimate load accurately by adjusting the load displacement time after time. I determine that concrete is poor, when Mises stress of any point reach $1.54 \mathrm{MPa}$, the standard values of concrete ultimate tensile strength. I determine that embedded hung part is tensile failure, if the stress of embedded hanging parts over yield stress $345 \mathrm{MPa}$ before concrete is poor.

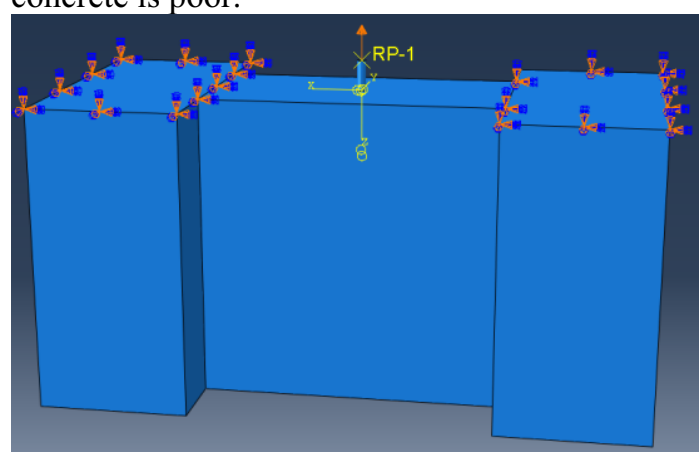

Figure 3. Loading and Constraint

\subsection{Results analysis}

\subsubsection{Stress nephogram of model}

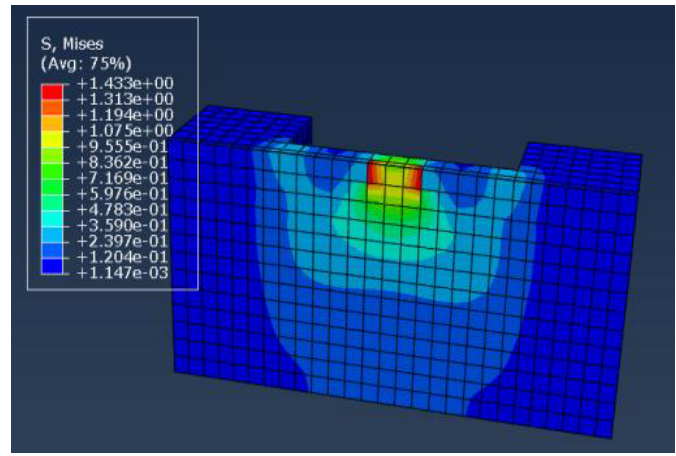

(a) Stress nephogram of YI

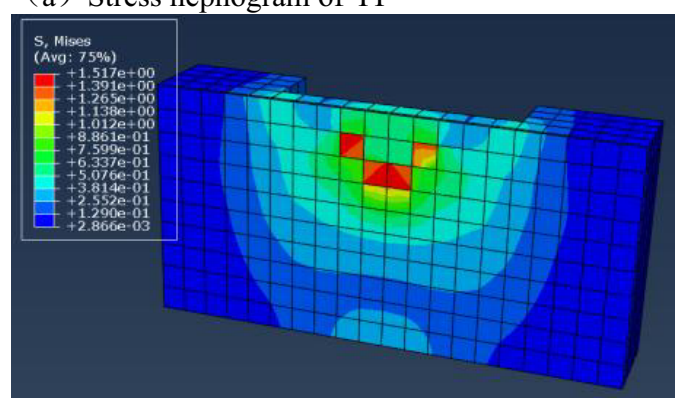

(b) Stress nephogram of Y2

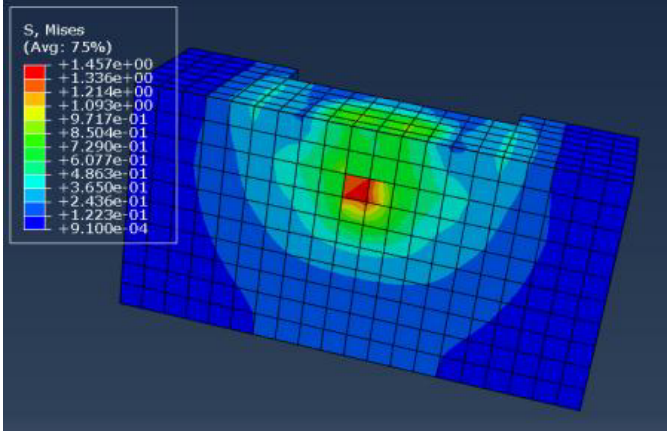

(c) Stress nephogram of Y3

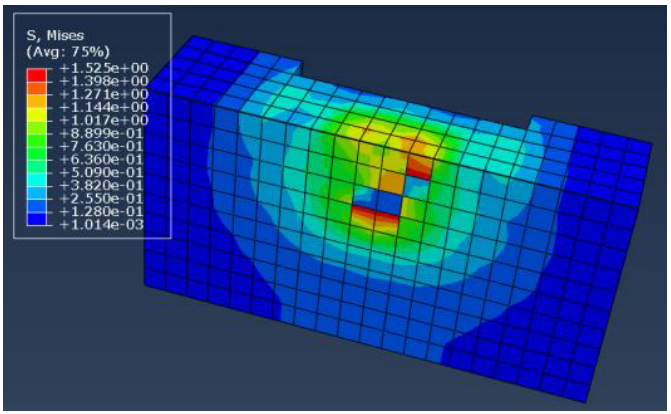

(d) Stress nephogram of Y4

Figure 4. Stress nephogram of models

As shown in figure 4, every models appear concrete vertebral failure result from the stress of concrete over ultimate tensile strength. Along the direction of burial depth, stress inside the concrete increase gradually, and the peak value appear at the bottom of embedded hanging parts. The stress inside the embedded hanging parts all less than yield stress, and maximal stress appear at the end of loading. Obviously, the damage radius of concrete vertebral body decrease gradually with the increasing of edge distance.

\subsubsection{Capacity - edge distance curve}

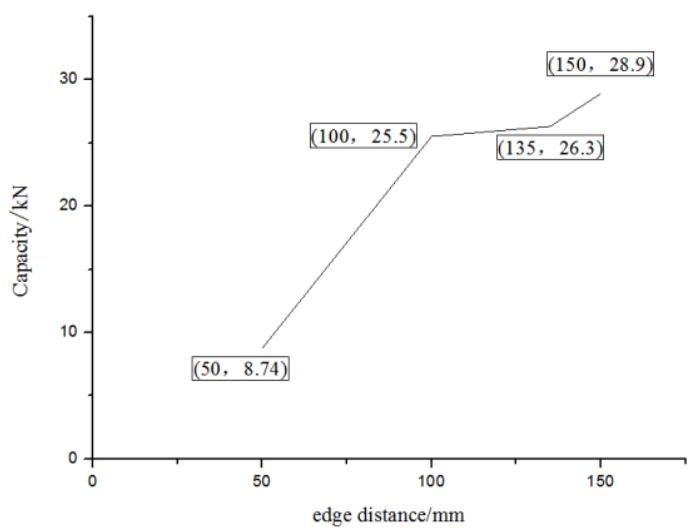

Figure 5. Capacity-edge distance curve

Table 2. Capacity summary
\begin{tabular}{|c|c|c|c|c|c|}
\hline Model number & Y1 & Y2 & Y3 & Y4 & Safety load \\
\hline Capacity (kN) & 8.74 & 25.5 & 26.3 & 28.9 & 22.5 \\
\hline
\end{tabular}

As shown in capacity-edge distance curve, the tensile bearing capacity of embedded hanging parts and the ultimate displacement increase gradually with the increasing of edge distance. The tensile bearing capacity increases slower when edge distance over critical value. 
As shown in Table 2, the safety load offered by producer less than the capacity of critical edge distance, but it has no enough safety factor.

\section{3 conclusion}

(1) The safety load greater than capacity of critical edge distance, indicating it doesn't apply to guiding the lifting and transporting of prefabricated part;

(2) The damage displacement of embedded hanging parts become smaller with decreasing the edge distance. And the greater the edge distance, the greater the capacity;

(3) The effect of edge distance to tensile bearing capacity of embedded hanging parts will be poor, when the edge distance more than critical value.

\section{Acknowledgments}

This work is financially supported by the Research Project of Urban and Rural Constriction system of Shen Yang (2011BAJ10B06)

\section{References}

1. Lin Zhi W, Dong Bin L, Fu Quan X. Earthquake Resistant Engineering and Retrofitting. J. 05, 101107(2013)

2. Tie Zhu J, Fu Quan X, Lin Zhi W. Earthquake Resistant Engineering and Retrofitting. J. 05, 108114(2013)

3. Lei S, Jie L, Zhou Dao L. Journal of Harbin Institute of Technology. J. 04,612-616(2010)

4. Gui Fang W, Building Structure. J.S1 ,436-438(2013)

5. Ting Peng Y, Zhou Dao L and Tao B. Structural Engineers. J. 02,97-100(2007) 